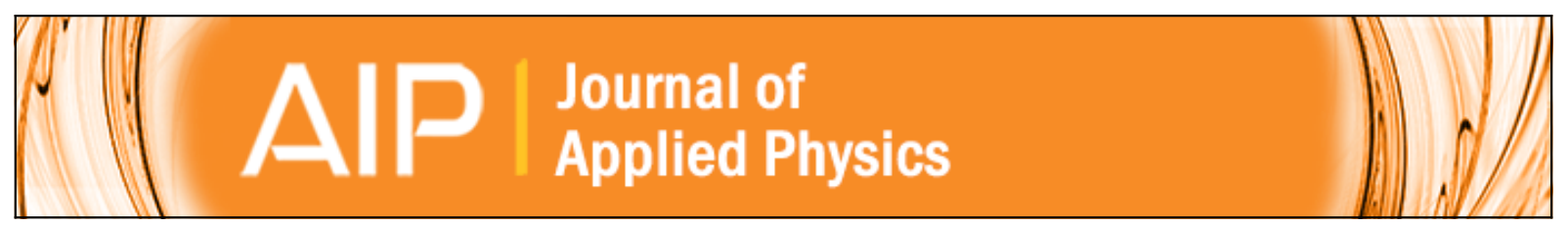

\title{
Ab initio study of shallow acceptors in bixbyite V2O3
}

N. Sarmadian, R. Saniz, B. Partoens, and D. Lamoen

Citation: Journal of Applied Physics 117, 015703 (2015); doi: 10.1063/1.4905316

View online: http://dx.doi.org/10.1063/1.4905316

View Table of Contents: http://scitation.aip.org/content/aip/journal/jap/117/1?ver=pdfcov

Published by the AIP Publishing

\section{Articles you may be interested in}

The electronic properties of point defects in earth-abundant photovoltaic material Zn3P2: A hybrid functional method study

J. Appl. Phys. 113, 013708 (2013); 10.1063/1.4772708

Mg doping in wurtzite $\mathrm{ZnO}$ coupled with native point defects: A mechanism for enhanced n-type conductivity and photoluminescence

Appl. Phys. Lett. 101, 042106 (2012); 10.1063/1.4738990

Donor-donor binding in In 203 : Engineering shallow donor levels

J. Appl. Phys. 107, 083704 (2010); 10.1063/1.3374644

Structural, electronic and magnetic properties of $\mathrm{V} 2 \mathrm{O} 5-\mathrm{x}$ : An ab initio study

J. Chem. Phys. 130, 214704 (2009); 10.1063/1.3146790

Ferromagnetism in Mg-doped AIN from ab initio study

Appl. Phys. Lett. 89, 142501 (2006); 10.1063/1.2358818

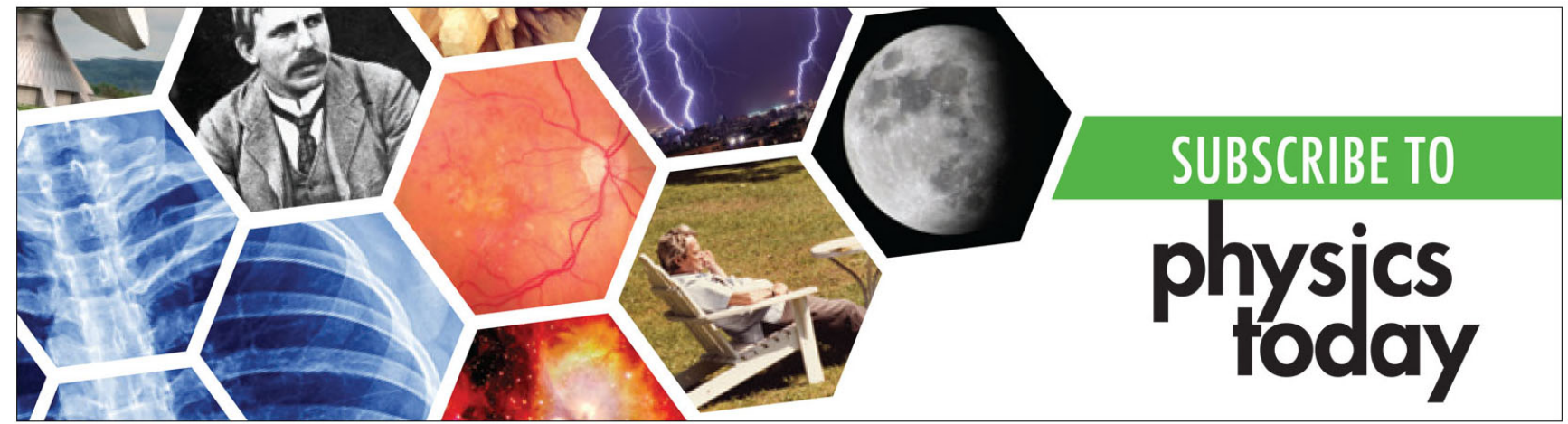




\title{
$A b$ initio study of shallow acceptors in bixbyite $\mathrm{V}_{\mathbf{2}} \mathrm{O}_{3}$
}

\author{
N. Sarmadian, ${ }^{\text {a) }}$ R. Saniz, B. Partoens, and D. Lamoen \\ CMT \& EMAT, Departement Fysica, Universiteit Antwerpen, Groenenborgerlaan 171, B-2020 Antwerpen, \\ Belgium
}

(Received 25 September 2014; accepted 19 December 2014; published online 5 January 2015)

\begin{abstract}
We present the results of our study on $p$-type dopability of bixbyite $\mathrm{V}_{2} \mathrm{O}_{3}$ using the Heyd, Scuseria, and Ernzerhof hybrid functional (HSE06) within the density functional theory (DFT) formalism. We study vanadium and oxygen vacancies as intrinsic defects and substitutional $\mathrm{Mg}, \mathrm{Sc}$, and $\mathrm{Y}$ as extrinsic defects. We find that $\mathrm{Mg}$ substituting $\mathrm{V}$ acts as a shallow acceptor, and that oxygen vacancies are electrically neutral. Hence, we predict $\mathrm{Mg}$-doped $\mathrm{V}_{2} \mathrm{O}_{3}$ to be a $p$-type conductor. Our results also show that vanadium vacancies are relatively shallow, with a binding energy of $0.14 \mathrm{eV}$, so that they might also lead to $p$-type conductivity. (C) 2015 AIP Publishing LLC.
\end{abstract}

[http://dx.doi.org/10.1063/1.4905316]

\section{INTRODUCTION}

There is continued interest in the semiconductor research community in novel $p$-type conductors. This is motivated by the desire of improving the properties of the materials currently used in electronic components such as light emitting diodes, transistors, or photovoltaic cells, or by the need of finding alternative materials to those already used, for reasons of cost or ease of synthesis.

Recently, we performed a DFT-based high-throughput screening of the properties of oxides with the bixbyite structure. In this study we found that $\mathrm{V}_{2} \mathrm{O}_{3}$ presents very good p-type dopability. ${ }^{1}$ This called for a more detailed study of this material, looking in particular for dopants resulting in possible $p$-type conductivity. Because of its unique properties, such as an intriguing metal-insulator transition, ${ }^{2}$ and its different applications in catalysts, chemical sensors, and cathode materials, $\mathrm{V}_{2} \mathrm{O}_{3}$ has been the subject of numerous investigations. ${ }^{3-5}$ The study of $\mathrm{V}_{2} \mathrm{O}_{3}$, however, has been mostly limited to its rhombohedral (corundum) and monoclinic structures. Vanadium sesquioxide was only recently synthesized in the bixbyite structure, ${ }^{6}$ and although several of its basic properties have now been reported, ${ }^{7,8}$ a more complete characterization requires further investigation.

In this work, we address specifically the prediction of $\mathrm{V}_{2} \mathrm{O}_{3}$ in the bixbyite structure as a novel $p$-type conductor by calculating the formation energies and charge transition levels of $\mathrm{V}$ and $\mathrm{O}$ vacancies as native defects and of elements $\mathrm{Mg}$, Y, and Sc substituting V as impurities.

In Sec. II, we explain the methodology used, and in Sec. III we present our results together with a discussion. We finish this work with Sec. III, where we summarize our main findings.

\section{COMPUTATIONAL METHOD}

We performed first-principles computations based on DFT, ${ }^{9,10}$ using the plane-wave Vienna Ab-initio Simulation Package (VASP). ${ }^{11,12}$ The projector augmented wave $(\mathrm{PAW})^{13,14}$ potentials are used to describe the electron-ion

\footnotetext{
a)nasrin.sarmadian@uantwerpen.be.
}

interactions. We use the HSE06 hybrid functional ${ }^{15,16}$ approximation to the exchange-correlation potential, both for structural relaxation and formation energy calculations. This is of particular importance in the present study, because the HSE06 functional is free of the band gap underestimation problem faced by other commonly used functionals and is capable of a reliable description of defect levels in semiconductors. ${ }^{17}$ Because of the computational cost of the HSE06 functional, we first perform our calculations on the primitive cell of the bixbyite structure, a body-centered cubic lattice (space group Ia $\overline{3}$, No. 206) with a basis containing 8 formula units, i.e., 40 atoms. Defects behaving as acceptors are then studied using the bixbyite conventional cell, containing 16 formula units, i.e., 80 atoms. An energy cutoff of $400 \mathrm{eV}$ was used for the plane-wave basis set. For structure relaxation and total energy calculations, the Brillouin zone of the primitive and conventional cells was sampled using a $3 \times 3 \times 3$, and a $2 \times 2 \times 2$ Monkhorst-Pack (MP) grid, respectively. ${ }^{18}$ Atomic relaxations were made until residual forces on the atoms were less than $0.01 \mathrm{eV} / \AA$ and total energies were converged to within $1 \mathrm{meV}$.

The bixbyite structure can be obtained from the fluorite structure by removing one fourth of the anion atoms, i.e., one fourth of the $\mathrm{O}$ atoms in the present case. As shown in Fig. 1, the $\mathrm{V}$ atoms in $\mathrm{V}_{2} \mathrm{O}_{3}$ occupy two inequivalent Wyckoff sites, $b$ (site symmetry S6) and $d$ (site symmetry $\mathrm{C} 2$ ), and have a sixfold $\mathrm{O}$ coordination in a distorted octahedral configuration (as opposed to the fluorite structure). Four of the $\mathrm{V}$ atoms occupy $b$ sites, and 12 occupy $d$ sites. This means that there are two possible inequivalent positions to substitute a $\mathrm{V}$ atom with a dopant atom. Note that a previous study shows that in the case of rare earth oxides with the bixbyite structure, if the substituting cation is larger than the host cation the dopant prefers the $b$-site, while the $d$-site is preferred if the substituting cation is instead smaller. ${ }^{19} \mathrm{We}$ also look into this question further down.

\section{RESULT}

By performing full optimization of the lattice constant and atomic positions using the HSE06 hybrid functional, we obtain a calculated lattice parameter of $9.287 \AA$. This is in 


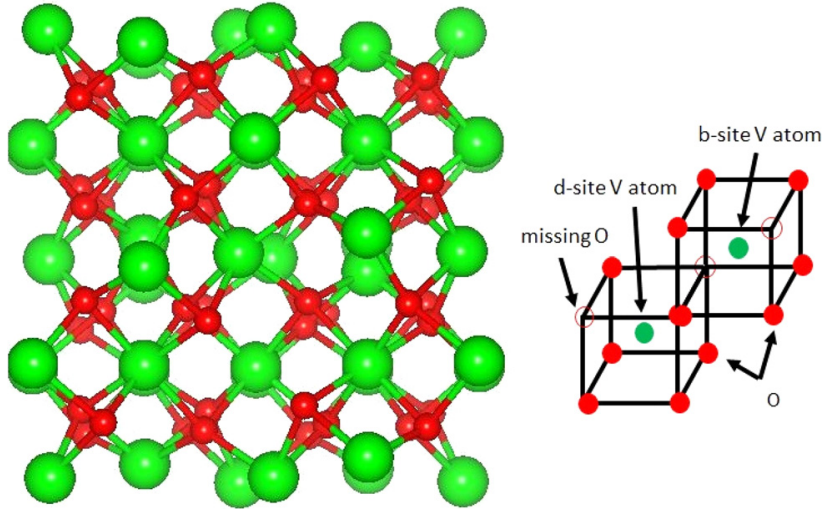

FIG. 1. Schematic illustration of atomic arrangements in V-O octahedra for $\mathrm{V}(\mathrm{b})$ and $\mathrm{V}(\mathrm{d})$ sublattices. The oxygen atoms are denoted by solid red circles, $\mathrm{V}$ atoms by green circles, and missing oxygen atoms by open circles.

very good agreement with the experimental value of $9.395 \AA$ A. ${ }^{6}$ First-principles calculations are reported in Refs. 6 and 7 , but the calculated lattice parameters are not indicated, so a comparison is not possible. We find $-10.43 \mathrm{eV}$ for the enthalpy of formation of $\mathrm{V}_{2} \mathrm{O}_{3}$ in its bixbyite structure, which is $0.07 \mathrm{eV}$ per formula unit higher than the enthalpy of formation we obtain for $\mathrm{V}_{2} \mathrm{O}_{3}$ in the corundum structure. This is in line with the findings in the first studies reporting bixbyite$\mathrm{V}_{2} \mathrm{O}_{3}$ as a metastable polymorph of vanadium sesquioxide. In those studies, it is found that the bixbyite phase is about $0.09 \mathrm{eV}$ per formula unit less stable than the corundum phase. ${ }^{6,7}$ It is also shown through quasiharmonic phonon calculations that the bixbyite phase is dynamically stable. ${ }^{6}$

Our HSE06 calculations indicate that bixbyite $\mathrm{V}_{2} \mathrm{O}_{3}$ is an indirect gap semiconductor, with the valence band maximum (VBM) at the $\Gamma$ point and the conduction band minimum $(\mathrm{CBM})$ at the $\mathrm{H}$ point, and a fundamental gap of $1.61 \mathrm{eV}$. The optical gap has a value of $1.98 \mathrm{eV}$ and is located at the $\mathrm{H}$ point, although the optical gap at other k-points is almost the same. Thus, the onset of optical absorption in this material should be rather clear in experiment. For some applications, such as light absorber layers, it is the optical gap that is relevant. Figure 2 shows the band structure of bixbyite $\mathrm{V}_{2} \mathrm{O}_{3}$ along high symmetry lines. Experimentally, the optical gap of bixbyite $\mathrm{V}_{2} \mathrm{O}_{3}$ colloidal nanocrystals has been reported in Ref. 8, where UV-vis spectroscopy absorbance indicates a direct gap of $1.29 \mathrm{eV}$.

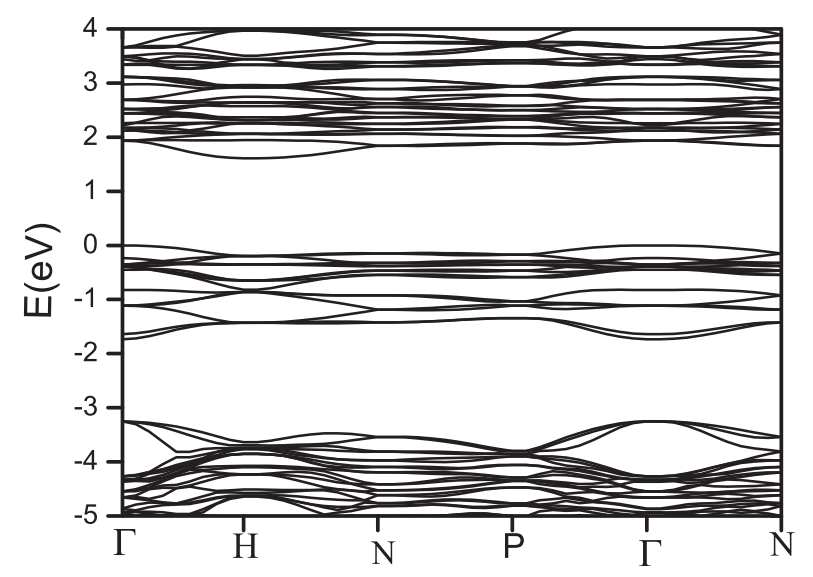

FIG. 2. HSE06 calculated Band structure of bixbyite $\mathrm{V}_{2} \mathrm{O}_{3}$.
There can be several reasons for the discrepancy between our result and experiment. In Ref. 8, it is indicated that the nanocrystals have a structure that is closer to the fluorite structure, due to the filling of the oxygen vacancies in the original bixbyite structure and present a uniform lattice expansion with respect to the latter. Hence, the observed gap in the nanocrystals may not be directly comparable to our calculated results because a lattice expansion usually results in a gap reduction, and the oxygen interstitials may give rise to impurity states in the gap, resulting again in an apparently smaller gap compared to the bulk bixbyite gap. On the other hand, it appears that the HSE06 functional tends to overestimate the band gap of vanadium oxides. This is the case for $\mathrm{VO}_{2}$ and $\mathrm{V}_{2} \mathrm{O}_{5},{ }^{20,21}$ and it might also be the case for $\mathrm{V}_{2} \mathrm{O}_{3}$, further contributing to the mismatch between our result and the reported value. However, it is important to recognize that the exact value of the band gap has very little bearing on the energy levels of the acceptor impurities we study in this work.

We note that the ground state structure of the bixbyite phase of $\mathrm{V}_{2} \mathrm{O}_{3}$ is found to be canted antiferromagnetic, with a phase transition to a paramagnetic phase around $50 \mathrm{~K}{ }^{6}$ Focusing on applications at room temperature, or at temperatures slightly above that, we can safely ignore magnetic effects in our calculations and consider bixbyite $\mathrm{V}_{2} \mathrm{O}_{3}$ as paramagnetic.

The mobility of the holes in a $p$-type material depends on the hole effective mass matrix. The bixbyite structure is cubic, so the corresponding matrix at the VBM is isotropic and it suffices to calculate the hole effective mass along one direction. We find a value of 6.807 along the $\Gamma-X$ direction. This value is somewhat high, but the high doping concentrations considered here can nevertheless lead to reasonably good conductivities.

The position of the CBM and VBM of a material with respect to its branch-point energy (BPE) can indicate whether it is $n$ - and/or $p$-type dopable. ${ }^{22}$ If the BPE falls high up in the band gap, or above the CBM, it is a good indication that the material is easily $n$-type dopable. Conversely, if the BPE falls low in the band gap or below the VBM, then the material will be easily doped $p$-type. The BPE can be calculated as a weighted average of the midgap energies over the Brillouin zone using the formula ${ }^{23,24}$

$$
E_{B P}=\frac{1}{2 N_{\mathbf{k}}} \sum_{\mathbf{k}}\left[\frac{1}{N_{C B}} \sum_{i}^{N_{C B}} \varepsilon_{c_{i}}(\mathbf{k})+\frac{1}{N_{V B}} \sum_{i}^{N_{V B}} \varepsilon_{v_{j}}(\mathbf{k})\right],
$$

where $\varepsilon_{c_{i}}(\mathbf{k})\left(\varepsilon_{v_{i}}(\mathbf{k})\right)$ is the energy of the $i$-th conduction (valence) band at point $\mathbf{k} . \mathrm{N}_{\mathbf{k}}$ is the number of points in the $\mathbf{k}$-point mesh, and $\mathrm{N}_{\mathrm{CB}}$ and $\mathrm{N}_{\mathrm{VB}}$ are the number of conduction and valence bands considered. The number of valence and conduction bands depends on the number of valence electrons in the primitive cell (excluding $d$ electrons) and is determined following the scaling rule introduced in the work of Schleife et al. ${ }^{23}$

In Fig. 3, on the left side, we plot the band edges of $\mathrm{V}_{2} \mathrm{O}_{3}$ with respect to the $\mathrm{BPE}$, setting the latter to $0 \mathrm{eV}$. One can readily see that it can be considered as a $p$-type dopable oxide. Hence, it should be possible to find dopants leading to 


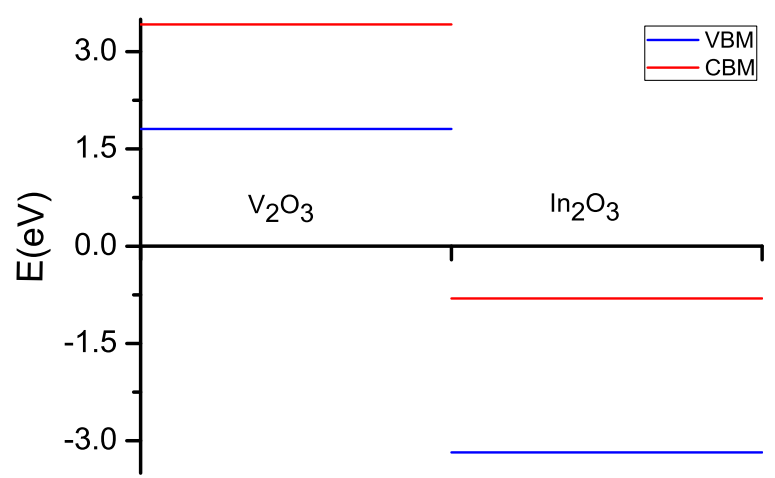

FIG. 3. Band alignment of $\mathrm{V}_{2} \mathrm{O}_{3}$ besides $\mathrm{In}_{2} \mathrm{O}_{3}$. ${ }^{33}$

shallow, or near shallow, acceptor levels in this oxide. For comparison, on the right side of Fig. 3 we present the alignment of the band edges of $\operatorname{In}_{2} \mathrm{O}_{3}$ with respect to the BPE, showing its well known $n$-type dopability character.

In general, $p$-type behavior may result from an intrinsic defect, such as a cation vacancy, or from an extrinsic defect, such as cation substitution with a foreign atom with less valence electrons. Indeed, this may result in a net electron deficiency and might give rise to acceptor levels. Thus, we consider first a vanadium vacancy, which we denote $V_{V}$. As extrinsic defect we consider $\mathrm{Mg}_{V}$, because the typical valence of $\mathrm{Mg}$ is $2+$, whereas $\mathrm{V}$ in $\mathrm{V}_{2} \mathrm{O}_{3}$ has in principle a valence $3+$. One can also consider substituting $\mathrm{V}$ with group 3 elements. Although the typical valence of the latter is also $3+$, the resulting isovalent center might behave as an acceptor, a donor, or remain electrically neutral. ${ }^{25}$ Hence, we consider the $\mathrm{Sc}_{V}$ and $\mathrm{Y}_{V}$ subsitutional defects in this study. Finally, it is important to consider $\mathrm{V}_{O}$, i.e., oxygen vacancy defects. Indeed, the latter may give rise to donor levels that could compensate the potential acceptor levels created by the previous defects, precluding any $p$-type behavior.

As indicated above, there are two inequivalent $\mathrm{V}$ positions in the bixbyite structure, and it is necessary to determine the preferred substitutional site. Table I compares the total energy of atoms $\mathrm{A}(\mathrm{A}=\mathrm{Mg}, \mathrm{Sc}$, and $\mathrm{Y})$ replacing $\mathrm{V}$ on the inequivalent $b$ - and $d$-sites. It shows that all three dopants prefer the $b$-site. Therefore, all formation energy calculations are performed with the substitutional dopants residing on this site. Table I also compares atomic radii for the three dopants and shows that $\mathrm{Mg}$ has a better lattice match than the other two.

TABLE I. Comparison of atomic radii (values from Ref. 26) and total energy for A-doped $\mathrm{V}_{2} \mathrm{O}_{3}(\mathrm{~A}=\mathrm{Sc}, \mathrm{Y}$, and $\mathrm{Mg})$ with $\mathrm{A}$ substituting $\mathrm{V}$ in the two possible inequivalent positions.

\begin{tabular}{lcc}
\hline \hline $\mathrm{A}$ & $\triangle R_{A, V}{ }^{\mathrm{a}}(\AA)$ & $\triangle E_{b, d}{ }^{\mathrm{b}}(\mathrm{eV})$ \\
\hline $\mathrm{Sc}$ & 0.25 & -0.18 \\
$\mathrm{Y}$ & 0.45 & -0.16 \\
$\mathrm{Mg}$ & 0.15 & -0.15 \\
\hline \hline
\end{tabular}

${ }^{\mathrm{a}} \triangle R_{A, V}=R_{A}-R_{V}$ where $R_{A}$, and $R_{V}$ are the atomic radius of substitutional impurity, and $\mathrm{V}$ atom, respectively.

${ }^{\mathrm{b}} \triangle E_{b, d}=E_{b}-E_{d}$ where $E_{b}$ and $E_{d}$ are the total energy of the defect system when the substitutional atom sits on the b-, and d-site, respectively.
As already mentioned, the formation energies of the defects are calculated using the HSE06 hybrid functional. For a defect $\mathrm{X}$ in charge state $\mathrm{q}$, the formation energy is given by ${ }^{27}$

$$
\begin{aligned}
E_{f}\left(X^{q}\right)= & E_{\text {tot }}\left(X^{q}\right)-E_{\text {tot }}(\text { bulk })-\sum_{i} n_{i} \mu_{i} \\
& +q\left(E_{F}+E_{v}+\Delta V\right),
\end{aligned}
$$

where $\mathrm{E}_{t o t}\left(\mathrm{X}^{q}\right)$ is the total energy of the cell containing the defect and $\mathrm{E}_{\text {tot }}$ (bulk) is the total energy of the pure $\mathrm{V}_{2} \mathrm{O}_{3} . n_{i}$ is the number of atoms of type $i$ added or removed from the cell $\left(n_{i}<0\right.$ if the atom is removed, and $n_{i}>0$ if the atom is added), and $\mu_{i}$ is the corresponding chemical potential. $\mathrm{E}_{F}$ is the Fermi energy, measured with respect to the VBM, $\mathrm{E}_{v}$, of the pure system and varies between zero and the gap value. $\Delta \mathrm{V}$ is the shift required to align the potentials in the undoped and doped cells for the purpose of band alignment. It was shown by Lyons and co-workers that the potential alignment can also correct the finite cell size effects on the formation energies of a charged defect, with an accuracy comparable to that of other methods. ${ }^{28}$ Here, we align the potentials in the defect and pure cells by calculating the $\Delta \mathrm{V}$ following the procedure used in Ref. 29.

The formation energies depend on the chemical potentials, which in turn depend on the experimental growth conditions. For $\mathrm{V}$ and $\mathrm{O}$, we take the limits imposed by the formation of $\mathrm{V}_{2} \mathrm{O}_{3}$ in the bixbyite phase. The $\mathrm{V}$ and $\mathrm{O}$ chemical potentials, $\mu_{\mathrm{V}}$ and $\mu_{\mathrm{O}}$, respectively, are mutually dependent through $E_{\text {tot }}($ bulk $)=2 \mu_{\mathrm{V}}+3 \mu_{\mathrm{O}}$. The upper limit of the $\mathrm{V}$ chemical potential, representing extreme V-rich conditions, is given by its elemental bulk phase, $\mu_{\mathrm{V}}=\mu_{\mathrm{V}(\mathrm{bulk})}$. Likewise, the upper limit for the $\mathrm{O}$ chemical potential, representing extreme O-rich conditions, is given by molecular oxygen, $\mu_{\mathrm{O}}=\mu_{\mathrm{O}\left(\mathrm{O}_{2}\right)}$. For the dopants, the chemical potential of $\mathrm{Mg}$, Sc, or $\mathrm{Y}$ is limited by the formation of the corresponding oxide, i.e., $\mathrm{MgO}, \mathrm{Sc}_{2} \mathrm{O}_{3}$, or $\mathrm{Y}_{2} \mathrm{O}_{3}$, respectively. Thus, for instance, the chemical potential of $\mathrm{Mg}$ is determined by $E_{\text {tot }}(\mathrm{MgO}$ bulk $)=\mu_{\mathrm{Mg}}+\mu_{\mathrm{O}}$. A corresponding expression applies to Sc and $\mathrm{Y}$.

Fig. 4 plots the formation energy of the most stable charge states of the intrinsic and extrinsic defects considered in $\mathrm{V}_{2} \mathrm{O}_{3}$ as a function of the Fermi energy $\mathrm{E}_{F}$ for both V-rich (Fig. 4(a)) and O-rich (Fig. 4(b)) conditions. One can see immediately that $\mathrm{Y}_{V}$ and $\mathrm{Sc}_{V}$ are very deep donors, with a transition level from charge state + to $0, \epsilon(+/ 0)$, lying low in the band gap. Thus, in both cases isovalent doping results in an essentially electrically neutral impurity. Note that the oxygen vacancy $\left(\mathrm{V}_{O}\right)$, which is a potential compensating native defect, has a high formation energy and occurs in its neutral state. Hence, it does not pose a problem to any possible $p$-type behavior.

The defects of interest to our study are $\mathrm{V}_{V}$ and $\mathrm{Mg}_{V}$, which present transition levels $\epsilon(0 /-2)=0.19 \mathrm{eV}$ and $\epsilon(0 /-)=0.17 \mathrm{eV}$, respectively, above the VBM, and thus act as acceptors. The question is whether these acceptors are shallow enough to give rise to $p$-type conductivity at, e.g., room temperature. For this, we study these defects in the bixbyite conventional 80-atom cell. This represents an 

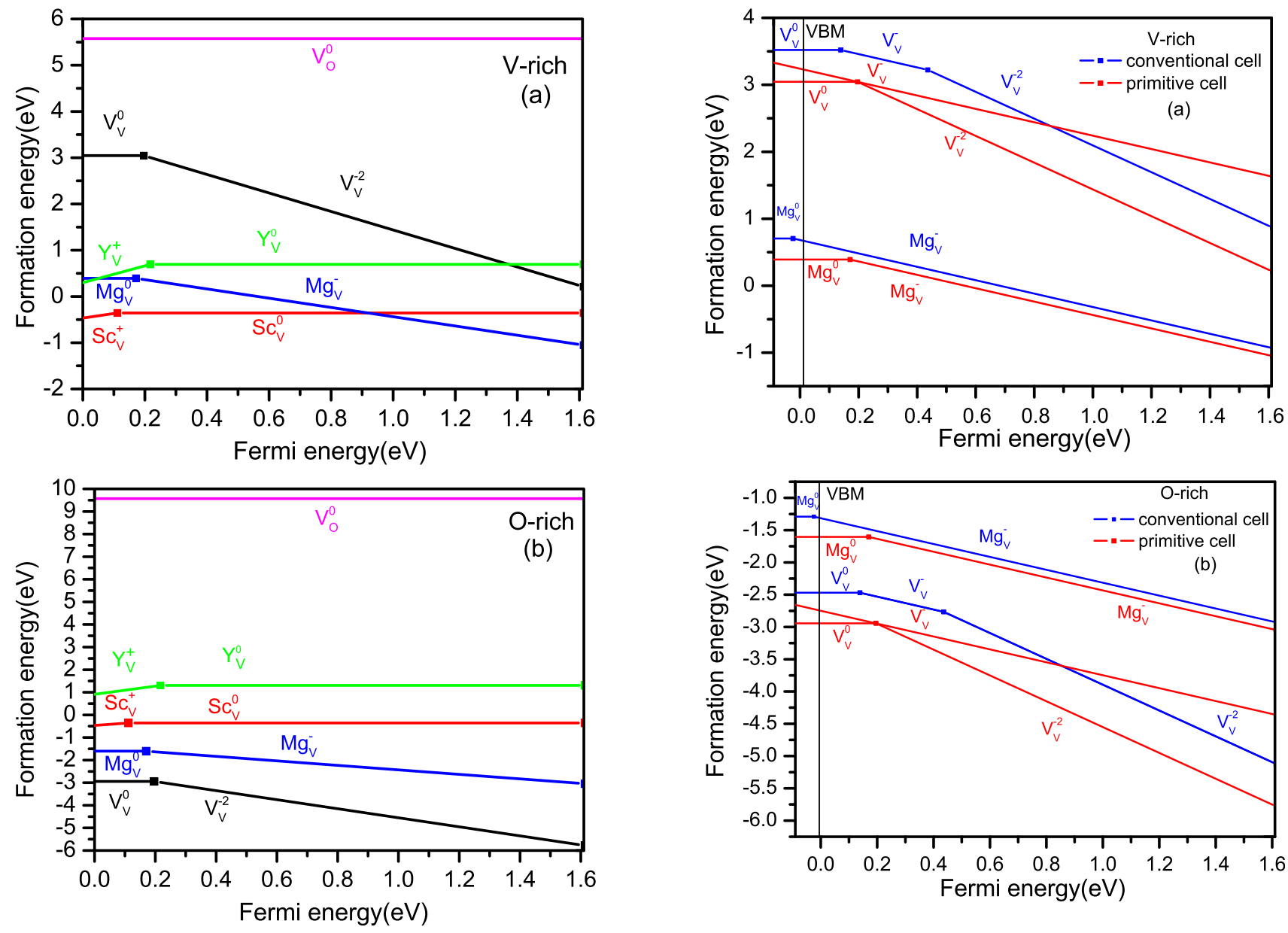

FIG. 4. Calculated intrinsic defect and impurity formation energies as a function of the Fermi energy under (a) V-rich, and (b) O-rich conditions. The calculations are performed for the primitive cell of $\mathrm{V}_{2} \mathrm{O}_{3}$ using the HSE06 functional.

impurity concentration of 1.25 at. $\%$, which corresponds to the typical concentrations in $n$ - and $p$-type transparent conducting oxides. ${ }^{30,31}$ In Fig. 5, we compare the transition levels of these two defects in the primitive and conventional bixbyite cells, both in V-rich [Fig. 5(a)] and O-rich [Fig. 5(b)] conditions. We immediately see that $\mathrm{Mg}_{V}$ becomes a shallow acceptor at this concentration, with the $\epsilon(0 /-)$ lying below the VBM. Furthermore, its formation energies remain low, while those of $\mathrm{V}_{O}$ (not shown) remain high. Thus, we predict $\mathrm{Mg}$-doped $\mathrm{V}_{2} \mathrm{O}_{3}$ to be a stable $p$-type conductor. The behavior of the $\mathrm{V}_{V}$ defect is somewhat different. In the bixbyite primitive cell, the $\epsilon(0 /-)$ transition level of this defect (not seen in Fig. 4, shown in Fig. 5) is slightly above the $\epsilon(0 /-2)$ transition level, with both transition levels almost touching. As shown in Fig. 5, in the conventional cell $\epsilon(0 /-)$ lies clearly below $\epsilon(0 /-2)$, at a energy $0.14 \mathrm{eV}$ above the VBM. Thus, as an acceptor, $\mathrm{V}_{V}$ is shallower in the conventional cell by $0.05 \mathrm{eV}$ compared to the primitive cell.

For a more complete description of the system studied, Fig. 6 plots the projected density of states (PDOS) for $\mathrm{V}_{2} \mathrm{O}_{3}$ and $\mathrm{Mg}$-doped $\mathrm{V}_{2} \mathrm{O}_{3}$. From Fig. 6(a), it can be seen that the character of the VBM and CBM of $\mathrm{V}_{2} \mathrm{O}_{3}$ is $V_{e_{g}}$, and $\mathrm{V}_{t_{28}}$, respectively. Note that the fact that the VBM and CBM consists of V $3 \mathrm{~d}$ states appears to be typical of $\mathrm{V}_{2} \mathrm{O}_{3}$, occurring

FIG. 5. The effect of diluting the defect concentration on the position of the transition level. The calculated formation energies of $\mathrm{Mg}_{V}$ and $\mathrm{V}_{V}$ as a function of the Fermi energy under (a) V-rich, and (b) O-rich conditions with HSE06.

also in its other phases. This is thought to be linked to its Mott-Hubbard insulator behavior at low temperature. ${ }^{32}$ In Mg-doped $\mathrm{V}_{2} \mathrm{O}_{3}$, the Fermi level lies below the VBM, as expected. This leads to the empty states seen as a small shoulder with partial $\mathrm{Mg}$ character just above the Fermi level.

Finally, Table II summarizes the cation $-O$ bond lengths in each defect system before and after optimization. Fig. 7 shows the relaxed positions of the $\mathrm{V}_{V}$ and $\mathrm{Mg}_{V}$ in $\mathrm{V}_{2} \mathrm{O}_{3}$. All substitutional atoms prefer to sit at the b-site (cf. Table I), where, because of symmetry, the A-O bond lengths of all six nearest neighbors are the same. Hence, there is only one bond length parameter for the nearest neighbors of each dopant. In the unrelaxed structure, the bond length is $2.00 \AA$. After optimization of the atomic positions, the bond length changes. Comparing Table II and Table I, we can see that by increasing the atomic radius of atom $\mathrm{A}$ (from $\mathrm{Mg}$ to Sc and then to Y), the A-O bond length increases.

\section{CONCLUSION}

We report on the electronic structure and defect properties of $\mathrm{V}_{2} \mathrm{O}_{3}$ as a novel $p$-type conductor. We study $\mathrm{Mg}, \mathrm{Y}$, and $\mathrm{Sc}$ as impurities substituting $\mathrm{V}$, as well as oxygen and vanadium vacancies as native defects. Our DFT calculations 

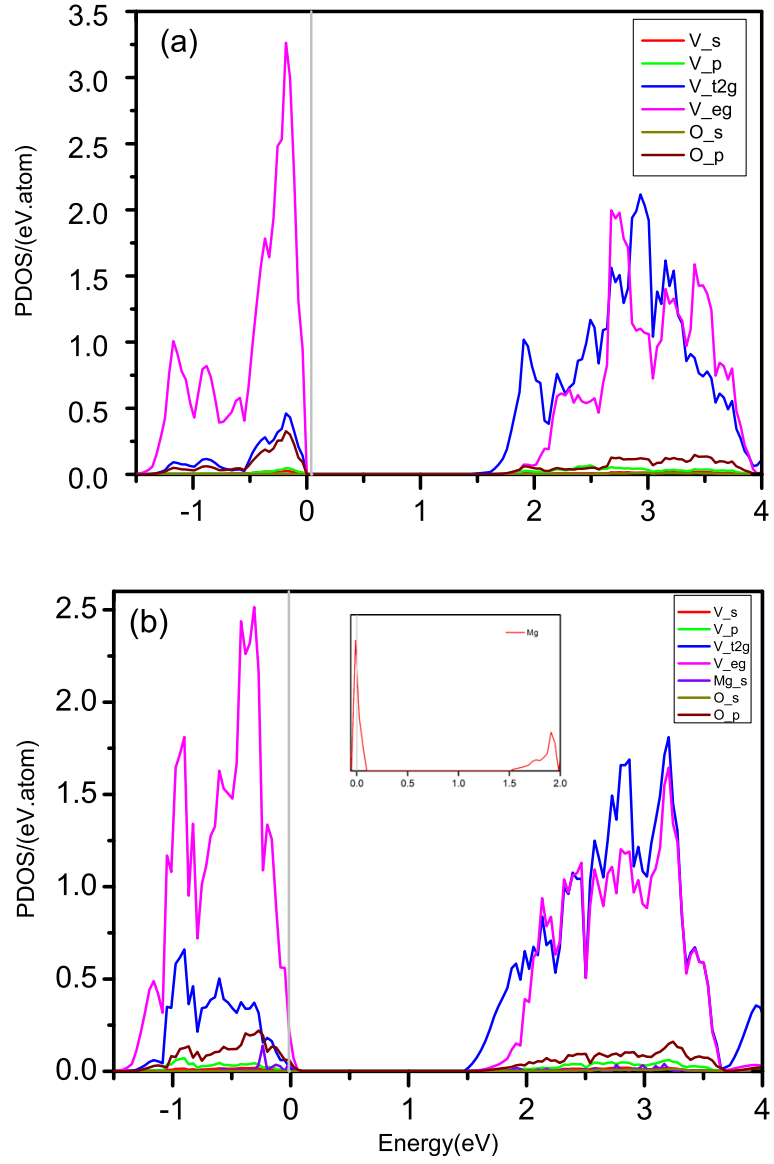

FIG. 6. HSE06 calculated PDOS of (a) pure $\mathrm{V}_{2} \mathrm{O}_{3}$, and (b) $\mathrm{Mg}$ doped $\mathrm{V}_{2} \mathrm{O}_{3}$ for primitive bixbyite cell.

TABLE II. Cation-O bond lengths ( $\AA$ ) for nearest neighbors in case of defective $\mathrm{V}_{2} \mathrm{O}_{3}$ compared with the $2.00 \AA$ bond length between $\mathrm{b}$-site $\mathrm{V}$ and $\mathrm{O}$.

\begin{tabular}{lc}
\hline \hline Site & Bond length \\
\hline $\mathrm{Sc}_{V}$ & 2.07 \\
$\mathrm{Y}_{V}$ & 2.21 \\
$\mathrm{Mg}_{V}$ & 2.05 \\
$\mathrm{~V}_{V}$ & 2.21 \\
\hline \hline
\end{tabular}

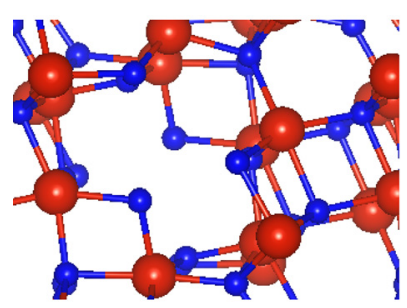

(a)

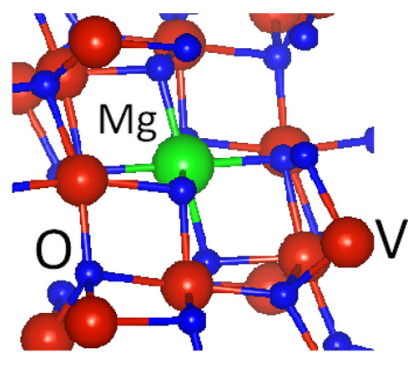

(b)
FIG. 7. Relaxed atomic geometries for (a) V vacancy, and (b) substitutional $\mathrm{Mg}$ in bixbyite $\mathrm{V}_{2} \mathrm{O}_{3}$. Red, blue, and green spheres show $\mathrm{V}, \mathrm{O}$, and $\mathrm{Mg}$ atoms, respectively.

show that, as in other bixbyite oxides, dopants with an atomic radius larger than the one of $\mathrm{V}$ prefer to substitute it on the b-site, rather than the d-site. We show that $\mathrm{Mg}_{V}$ acts as a shallow acceptor, with a low formation energy.
Furthermore, we find that oxygen vacancies are electrically neutral, and hence do not behave as hole-killing defects. Thus, we predict $\mathrm{Mg}$-doped $\mathrm{V}_{2} \mathrm{O}_{3}$ to be a $p$-type conductor. We also show that $\mathrm{V}_{V}$ acts as a relatively shallow acceptor, with an activation energy of $0.14 \mathrm{eV}$. Thus, it may also lead to $p$-type conductivity. On the other hand, we find that substitutional $\mathrm{Sc}$ and $\mathrm{Y}\left(\mathrm{Sc}_{V}\right.$ and $\left.\mathrm{Y}_{V}\right)$ behave as deep donors.

\section{ACKNOWLEDGMENTS}

We gratefully acknowledge financial support from the FWO-Vlaanderen through Project No. G.0150.13 and a GOA fund from the University of Antwerp. The computational resources and services used in this work were provided by the VSC (Flemish Supercomputer Center) and the HPC infrastructure of the University of Antwerp (CalcUA), both funded by the Hercules Foundation and the Flemish Government-department EWI.

${ }^{1}$ N. Sarmadian, R. Saniz, B. Partoens, D. Lamoen, K. Volety, G. Huyberechts, and J. Paul, Phys. Chem. Chem. Phys. 16, 17724 (2014).

${ }^{2}$ A. Tanaka, Physica B 329, 753 (2003).

${ }^{3}$ M. Wu, X. Lin, Y. Wang, L. Wang, W. Guo, D. Qi, X. Peng, A. Hagfeldt, M. Grätzel, and T. Ma, J. Am. Chem. Soc. 134, 3419 (2012).

${ }^{4}$ A. Julbe, D. Farrusseng, and C. Guizard, Sep. Purif. Technol. 25, 11 (2001).

${ }^{5}$ Z. Xu, J. Luo, and K. T. Chuang, ECS Trans. 11, 1 (2008).

${ }^{6}$ D. Weber, A. Stork, S. Nakhal, C. Wessel, C. Reimann, W. Hermes, A. Müller, T. Ressler, R. Pöttgen, T. Bredow, R. Dronskowski, and M. Lerch, Inorg. Chem. 50, 6762 (2011).

${ }^{7}$ C. Wessel, C. Reimann, A. Müller, D. Weber, M. Lerch, T. Ressler, T. Bredow, and R. Dronskowski, J. Comput. Chem. 33, 2102 (2012).

${ }^{8}$ A. Bergerud, R. Buonsanti, J. L. Jordan-Sweet, and D. J. Milliron, Chem. Mater. 25, 3172 (2013).

${ }^{9}$ P. Hohenberg and W. Kohn, Phys. Rev. 136, B864 (1964).

${ }^{10}$ W. Kohn and L. J. Sham, Phys. Rev. 140, A1133 (1965).

${ }^{11}$ G. Kresse and J. Hafner, Phys. Rev. B 47, 558 (1993).

${ }^{12}$ G. Kresse and J. Furthmüller, Comput. Mater. Sci. 6, 15 (1996).

${ }^{13}$ G. Kresse and D. Joubert, Phys. Rev. B 59, 1758 (1999).

${ }^{14}$ P. E. Blöchl, Phys. Rev. B 50, 17953 (1994).

${ }^{15}$ J. Heyd, G. E. Scuseria, and M. Ernzerhof, J. Chem. Phys. 118, 8207 (2003).

${ }^{16}$ A. V. Krukau, O. A. Vydrov, A. F. Izmaylov, and G. E. Scuseria, J. Chem. Phys. 125, 224106 (2006).

${ }^{17}$ A. Alkauskas, P. Deák, J. Neugebauer, A. Pasquarello, and C. G. Van de Walle, Advanced Calculations for Defects in Materials: Electronic Structure Methods (Wiley-VCH, Weinheim, Germany, 2011).

${ }^{18}$ H. J. Monkhorst and J. D. Pack, Phys. Rev. B 13, 5188 (1976).

${ }^{19}$ C. R. Stanek, K. J. McClellan, B. P. Uberuaga, K. E. Sickafus, M. R. Levy, and R. W. Grimes, Phys. Rev. B 75, 134101 (2007).

${ }^{20}$ J. E. Coulter, E. Manousakis, and A. Gali, Phys. Rev. B 88, 041107 (2013).

${ }^{21}$ W. Li, C. F. J. Walther, A. Kuc, and T. Heine, J. Chem. Theory Comput. 9, 2950 (2013).

${ }^{22}$ A. Schleife, F. Fuchs, C. Rödl, J. Furthmüller, and F. Bechstedt, Appl. Phys. Lett. B 94, 012104 (2009).

${ }^{23}$ P. Deák, B. Aradi, and T. Frauenheim, J. Phys. Chem. C 115, 3443 (2011).

${ }^{24}$ B. Höffling, A. Schleife, F. Fuchs, C. Rödl, and F. Bechstedt, Appl. Phys. Lett. B 97, 032116 (2010).

${ }^{25}$ P. Y. Yu and M. Cardona, Fundamentals of Semiconductors (SpringerVerlag, Berlin, 2010).

${ }^{26}$ J. C. Slater, J. Chem. Phys. 41, 3199 (1964).

${ }^{27}$ H. J. Kim, U. Kim, T. H. Kim, J. Kim, H. M. Kim, B. G. Jeon, W. J. Lee, H. S. Mun, K. T. Hong, J. Yu, K. Char, and K. H. Kim, Phys. Rev. B 86, 165205 (2012).

${ }^{28}$ J. L. Lyons, A. Janotti, and C. G. Van de Walle, Phys. Rev. Lett. B 108, 156403 (2012).

${ }^{29}$ M. N. Amini, R. Saniz, D. Lamoen, and B. Partoens, J. Appl. Phys. 110, 063521 (2011).

${ }^{30}$ A. Stadler, Materials 5, 661 (2012). 
${ }^{31}$ D. Ginley, B. Roy, A. Ode, C. Warmsingh, Y. Yoshida, P. Parilla, C. Teplin, T. Kaydanova, A. Miedaner, C. Curtis, A. Martinson, T. Coutts, D. Readey, H. Hosono, and J. Perkins, Thin Solid Films 445, 193 (2003).

${ }^{32}$ Y. Guo, S. J. Clark, and J. Robertson, J. Chem. Phys. 140, 054702 (2014).
${ }^{33}$ Note that in Ref. 1 the amount of exact exchange used for the hybrid functional calculations is 0.325 , while in the present work we use the standard value of 0.25 . For this reason the band gaps and band alignment are somewhat different in that work. 